\title{
Aeromonas salmonicida Type I pilus system contributes to host colonization but not invasion
}

\author{
Andrew Dacanay ${ }^{1,2, *}$, Jessica M. Boyd ${ }^{1,3}$, Mark D. Fast ${ }^{1,4}$, Leah C. Knickle ${ }^{1}$, \\ Michael E. Reith ${ }^{1}$ \\ ${ }^{1}$ National Research Council of Canada Institute for Marine Biosciences, 1411 Oxford Street, Halifax, \\ Nova Scotia B3H 3Z1, Canada \\ ${ }^{2}$ Present address: Department of Plant and Animal Sciences, Nova Scotia Agricultural College, \\ PO Box 500, Truro, Nova Scotia B2N 5E3, Canada \\ ${ }^{3}$ Present address: Department of Biology, St. Mary's University, 923 Robie Street, Halifax, Nova Scotia B3H 3C3, Canada \\ ${ }^{4}$ Present address: School of Marine and Atmospheric Sciences, Stony Brook University, Stony Brook, \\ New York 11794-5000, USA
}

\begin{abstract}
The host-adherence strategies employed by Aeromonas salmonicida subsp. salmonicida, the etiological agent of an infectious bacteremia of salmonids, are poorly understood. In addition to the outer protein coat or S-layer, $A$. salmonicida has both Type I and Type IV pili loci. The $A$. salmonicida Type I or Fim pilus is encoded by an operon with genes for a chaperone, an usher, and 3 pilus subunits and is predicted to be similar to the Pap fimbriae of uropathogenic Escherichia coli, which are considered significant virulence factors. A Fim-deficient strain of $A$. salmonicida strain A449, $\Delta$ fim, was created by deleting this operon. Virulence of $\Delta$ fim was unchanged in direct live challenges of Atlantic salmon Salmo salar L., a natural host for A. salmonicida. A measure of clinically inapparent (covert) infections suggested Fim was required to establish or maintain a covert infection. This was confirmed by an ex vivo adherence and invasion assay using freshly excised salmon gastrointestinal (GI) tract, which showed that, compared to the parental strain, the ability of the isogenic $\Delta$ fim mutant strain to adhere to the salmon GI tract was reduced but, once adhered, its ability to invade was unchanged. Thus the Fim pilus functions as an adhesin in A. salmonicida and the presence of a functional Fim improved the efficiency of A. salmonicida infection of Atlantic salmon.
\end{abstract}

KEY WORDS: Pilin · Adhesin · Furunculosis · Virulence

\section{INTRODUCTION}

Aeromonas salmonicida subsp. salmonicida (hereinafter referred to as A. salmonicida) is the etiological agent of an infectious bateriemia of Atlantic salmon called furunculosis. While A. salmonicida is transmitted horizontally through the water column, little is understood about the strategies it uses to colonize the host during infection. Furunculosis presents as a spectrum of diseases depending on the age and health status of the salmon. Acute and per-acute infections are characterized by high mortality and morbidity rates and affected animals show few or no clinical signs except for ecchymosis of the swim bladder. Low morbidity rates are seen in sub-acute and chronic infections where affected animals display the characteristic, focal dermomyonecrotic lesions or furuncles that are considered pathognomonic for the disease. The clinically inapparent, or covert, infection state is characterized by no morbidity or mortality and an outwardly healthy appearance. A disease state is manifested when the salmon is placed under acute or chronic stress. Such conditions temporarily immunosuppress the salmon, allowing the covert infection to become overt.

Recent work (Jutfelt et al. 2008) indicates that live Aeromonas salmonicida can cross the salmon intestinal 
epithelium by a trans-cellular route and that this translocation requires bacteria with intact and functional surface structures. Our continued interest in $A$. salmonicida adhesins leads us to ask if we could identify factors important for adherence to salmon intestinal tissue.

The most well studied Aeromonas salmonicida adhesin is its outer protein coat, the S-layer, the major protein constituent of which is VapA. While the S-layer has long been demonstrated to be both an important virulence factor and an adhesin (Ishiguro et al. 1981, Trust et al. 1983, Garduño et al. 1992, Kay \& Trust 1997, Garduño et al. 2000), VapA-deficient strains may still be infectious (Ellis et al. 1988, Olivier 1990). In addition to the S-layer, A. salmonicida strain A449 also has genes for at least 4 specific adhesion systems: Tap, Flp, MSHA, and Fim (Reith et al. 2008). The first 3 are Type IV pili and have been characterized elsewhere (Masada et al. 2002, Boyd et al. 2008). The latter system, Fim, is predicted to encode a Type I pilus, similar to the fimbriae of pathogenic Escherichia coli that are considered to be important virulence factors in that organism. The present study reports on the contribution of Fim to A. salmonicida strain A449 virulence as assessed by a Fim-deficient isogenic laboratory strain.

\section{MATERIALS AND METHODS}

Bacterial strains and growth conditions. Bacteria and plasmids used in the present study are listed in Table 1. The parental strain for all knockouts was Aeromonas salmonicida subsp. salmonicida strain A449 (hereinafter A449) originally isolated from a natural furunculosis epizootic in brown trout Salmo trutta (Michel 1979). All A. salmonicida strains were grown in tryptic soy broth (TSB) or agar (TSA) (Difco) for $3 \mathrm{~d}$ at $17^{\circ} \mathrm{C}$ with shaking. Escherichia coli strains were grown in Luria-Bertani broth (LB) or agar at $37^{\circ} \mathrm{C}$. Antibiotics were used at the following concentrations:
E. coli: ampicillin, $100 \mu \mathrm{g} \mathrm{m} \mathrm{m}^{-1}$ and kanamycin, $25 \mu \mathrm{g}$ $\mathrm{ml}^{-1}$; A. salmonicida: ampicillin, $50 \mu \mathrm{g} \mathrm{ml}^{-1}$ and chloramphenicol, $20 \mu \mathrm{g} \mathrm{ml}^{-1}$.

Mutant construction. An in-frame, unmarked deletion of the entire fim locus, hereinafter referred to as $\Delta$ fim, was created using crossover PCR (Link et al. 1997). Briefly, 2 self-complementary PCR fragments of ca. 500 bp flanking the fim locus were amplified from A449 chromosomal DNA using the primers shown in Table 2. The 2 PCR fragments were mixed together and amplified with the 2 external (Fim-No and FimCo) primers to generate a ca. $1 \mathrm{kbp}$ fragment carrying DNA flanking the fimA and fimF genes. The joined fragments were cloned into the mobilizable, pirdependent, sucrase-expressing vector pWM91 to generate pWM-fim, which was conjugated into A449 from Escherichia coli BW20767. Single-crossover integrants were selected by plating on TSA supplemented with ampicillin to select for integrants and chloramphenicol to select against E. coli. Double-crossover segregants were isolated by selection on TSA with $15 \%$ sucrose to select against plasmid-containing colonies. Correct deletion of the fim locus was determined by PCR with primers complementary to the chromosome outside of the flanking regions and with primers complementary to the fimC gene. This resulted in the creation of a small open reading frame encoding the first 8 amino acids (aa) of FimA and the last 17 aa of FimF connected by the 7 aa of the crossover tag.

Bacterial conjugation. Escherichia coli BW20767 carrying pWM-fim was grown in LB with ampicillin overnight at $37^{\circ} \mathrm{C}$. A449 was grown in TSB with chloramphenicol for $3 \mathrm{~d}$ at $17^{\circ} \mathrm{C}$. One $\mathrm{ml}$ of each bacterial species was harvested and washed twice in fresh TSB without antibiotics. After the last wash, the bacterial pellet was resuspended in a small volume (15 to $30 \mu \mathrm{l})$ of TSB. These thick suspensions of each bacterium were mixed together thoroughly and spotted in the center of a TSA plate without antibiotics. After $2 \mathrm{~d}$ of incubation at $17^{\circ} \mathrm{C}$, the spot was removed with a sterile

Table 1. Bacterial strains and plasmids used. $\mathrm{Cm}^{\mathrm{R}}$ : chloramphenicol resistant; $\mathrm{Ap}^{\mathrm{R}}$ : ampicillin resistant

\begin{tabular}{|c|c|c|}
\hline Strain or plasmid & Description & Source \\
\hline \multicolumn{3}{|c|}{ Aeromonas salmonicida } \\
\hline A449 & A. salmonicida subsp. salmonicida isolate, $\mathrm{Cm}^{\mathrm{R}}$ & Michel (1979) \\
\hline$\Delta$ fim & $\mathrm{A} 449 \Delta$ fim, $\mathrm{Cm}^{\mathrm{R}}$ & Present study \\
\hline \multicolumn{3}{|c|}{ Escherichia coli strains } \\
\hline TOP10 & K-12 & Invitrogen \\
\hline EC100D pir-116 & K-12, pir-116 & Epicenter \\
\hline BW20767 & K-12, pir+, conjugation+ & Metcalf et al. (1996) \\
\hline \multicolumn{3}{|l|}{ Plasmids } \\
\hline pWM91 & 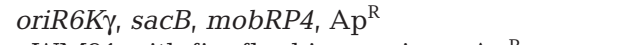 & Metcalf et al. (1996) \\
\hline pWM-fim & pWM91 with fim flanking regions, $\mathrm{Ap}^{\mathrm{R}}$ & Present study \\
\hline
\end{tabular}


Table 2. Oligonucleotides used. Restriction sites are underlined, non-sequence-specific regions in lower case

\begin{tabular}{|c|c|c|}
\hline Primer name & Sequence & Purpose \\
\hline Fim-No & tgtgctcgagCGTGCTCAATGTTGGTTTTT & $\begin{array}{l}\text { To amplify } 5 \text { ' flanking region for knockout, } \\
\text { carries XhoI site }\end{array}$ \\
\hline Fim-Ni & cccatccactaaacttaaacaAACCAAAATTTTATTAGCGTTCATTC & $\begin{array}{l}\text { To amplify 5' flanking region for knockout, } \\
\text { carries crossover sequence }\end{array}$ \\
\hline Fim-Ci & tgtttaagtttagtggatgggATCAAACCTGGCGATTATACCG & $\begin{array}{l}\text { To amplify } 3 \text { ' flanking region for knockout, } \\
\text { carries crossover sequence }\end{array}$ \\
\hline Fim-Co & atatcacgatgcggccgcGGGAGAGAATGTTTCCCACA & $\begin{array}{l}\text { To amplify } 3 \text { ' flanking region for knockout, } \\
\text { carries NotI site }\end{array}$ \\
\hline Fim-UF1 & AATCCTGATTGCCTGATTGC & To verify deletion, flanks deletion on left side \\
\hline Fim-DR1 & ССАТТСТСАTCGGAAAGGAA & To verify deletion, flanks deletion on right side \\
\hline Fim-F1 & GCTGACTTGACCCAATCCAT & To verify deletion, within deletion \\
\hline Fim-R1 & TATCAAGATTACAGCCGGCC & To verify deletion, within deletion \\
\hline
\end{tabular}

toothpick and resuspended in $1 \mathrm{ml}$ TSB, diluted, and plated on TSA with the appropriate antibiotics. Large colonies were picked, streaked to fresh plates, and passaged 2 more times.

Animal care. Both the National Research Council Halifax's Local Animal Care Committee and Dalhousie University's Committee on Laboratory Animals approved all animal procedures, which were conducted under Canadian Council on Animal Care guidelines. Under these guidelines death is considered an unacceptable endpoint (Demers et al. 2006; www.ccac.ca). Moribund animals were euthanized when a previously agreed set of limiting clinical signs were reached. Data are thus reported as cumulative morbidity and mean time to morbidity (MTTM) to better reflect this reality.

Juvenile St. John River stock Atlantic salmon (Salmo salar L. 1758), ca. 120 g, were obtained from a Nova Scotia hatchery certified under Canadian Fish Health Protection Regulations. They were stocked in 1001 fiberglass resident tanks at a stocking density of ca. $2.4 \mathrm{~kg} 100 \mathrm{l}^{-1}$ and maintained at $14 \pm 2{ }^{\circ} \mathrm{C}$, in flowthrough in dechlorinated municipal (fresh) water. They were fed a maintenance ration daily of $1 \%$ bodyweight of a commercially available feed (Signature Salmon Ration, Shurgain). Feeding was suspended for $1 \mathrm{~d}$ prior to and $1 \mathrm{~d}$ post-manipulation.

Challenge. The virulence of the mutant strain $\Delta$ fim and its parental strain A449 were tested by intraperitoneal (i.p.) and immersion challenges conducted as per Dacanay et al. (2006). Briefly, for the immersion challenge there were 2 tanks group ${ }^{-1}$ with ca. 40 fish $\operatorname{tank}^{-1}$. The fish were transferred into commercially available plastic containers, sedated (15 $\mathrm{mg} \mathrm{l}^{-1}$ tricaine methanosulphonate [TMS]; Syndel Laboratories), and exposed for $40 \mathrm{~min}$ to ca. $10^{6} \mathrm{cfu} \mathrm{ml}^{-1}$ of the appropriate strain before being returned to their designated resident tanks. Control fish experienced identical handling but were only exposed to an equal volume of phosphate-buffered saline (PBS). For i.p. challenge, there were 2 tanks per group with ca. 30 fish per tank. They were anesthetized with $50 \mathrm{mg} \mathrm{l}^{-1}$ TMS prior to injection with ca. $10^{4} \mathrm{cfu}_{\text {animal }}{ }^{-1}$ of the appropriate strain in $100 \mu \mathrm{l} \mathrm{PBS}$. Control animals were injected with an equal volume of PBS. Doses were confirmed retrospectively by direct colony counts on TSA supplemented with $20 \mu \mathrm{g} \mathrm{ml}^{-1}$ chloramphenicol.

Animals were closely monitored post-challenge. Morbid animals were euthanized with a TMS overdose once they had met the minimum criteria for euthanasia. The posterior kidney was sampled aseptically onto TSA as recommended by the American Fisheries Society (Schotts 1994). The TSA was supplemented with $20 \mu \mathrm{g} \mathrm{ml}^{-1}$ chloramphenicol.

Statistical significance in cumulative morbidity between groups was assessed by the G-test, a modified $\chi^{2}$, which was calculated manually. Statistical significance in MTTM between groups was assessed by $t$-test and was calculated by GraphPad Prism 5.0. Significance was set as $\mathrm{p}<0.05$ for both tests.

Stress test. It has been long known that Aeromonas salmonicida can induce a clinically inapparent or covert infection state (Hiney et al. 1997) and that adhesins such as Fim may play an important role in establishing such a state. Sixty days after exposure by immersion, covert infection levels in the remaining animals were assessed using a modified stress test (Specker et al. 1994). Briefly, the animals were anesthetized as before (see 'Challenge' above) and $100 \mathrm{mg}$ $\mathrm{kg}^{-1}$ cortisol (hydrocortisone; Sigma-Aldrich) emulsion in 1:1 vegetable oil:vegetable fat was administered by i.p. injection. The animals were returned to their resident tank and monitored for $10 \mathrm{~d}$, after which they were euthanized. To ensure sufficient numbers of animals for statistics, animals from both A449 and $\Delta$ fim tanks were combined to give a total number of 40 animals for both. Statistical significance in cumulative morbidity and MTTM between groups was assessed as for the challenges. 
Ex vivo adherence. An ex vivo assay was performed to determine if the $\Delta$ fim strain had reduced host-adherence abilities. The gastrointestinal (GI) tract was chosen as the substrate against which bacterial adherence would be tested as it could be readily collected and manipulated in a sterile manner. Hindgut was sterilely excised from large (>2 kg) Atlantic salmon following euthanasia with an overdose of anesthetic (>100 $\mathrm{mg} \mathrm{l}^{-1}$ TMS). Gut was prepared by first cutting the tube of gut open sagittally, followed by cutting it axially to produce rectangles of ca. $100 \mathrm{~mm}^{2}$. Feces were removed with sterile forceps and by washing in sterile PBS ( $\mathrm{pH}$ 7.4). The pieces of gut were placed on sterile TSA and $10 \mu \mathrm{l}$ of an $\mathrm{OD}_{600}$ 0.001 solution of either $\Delta$ fim or the parental strain A449 was added to the lumenal side of the gut square. The bacteria and gut were incubated for $2 \mathrm{~h}$ at room temperature. After this time non-adherent bacteria were removed by gently washing the gut square with sterile PBS. Adherent bacteria were removed by vigorous vortexing in PBS and subsequently enumerated by direct colony counts on TSA supplemented with $20 \mu \mathrm{g} \mathrm{ml}^{-1}$ chloramphenicol. After removal of the adherent bacteria, gut squares were placed in Liebowitz-15 (L-15) medium (SigmaAldrich) supplemented with $21.5 \mu \mathrm{g} \mathrm{ml}^{-1}$ gentamicin (Gibco) and incubated for a further $2 \mathrm{~h}$ at room temperature to kill extracellular bacteria. Invasive bacteria were enumerated following hypotonic shock: gut squares were transferred into a lysis solution (sterile $0.1 \%$ Tween 20 in $\mathrm{ddH}_{2} \mathrm{O}$ ) and vortexed vigorously. Bacterial titer was determined by direct colony counts as before.

The area of the gut squares was measured and the bacterial titer normalized per $\mathrm{mm}^{2}$. Bacteria adherent after $2 \mathrm{~h}$ are reported as a percentage of the original innoculum. Invasive bacteria are reported as a percentage of the adherent bacteria. Statistical differences in adherence or invasion were determined by $t$-test (GraphPad Prism 5.00); significance was set at $\mathrm{p}<0.05$.

\section{RESULTS}

\section{Gene identification}

A region of the Aeromonas salmonicida strain A499 genome (Reith et al. 2008) was identified that contained 6 genes predicted to encode a Type I or chaperone/usher type pilus (Table 3 ). The proteins encoded by these genes are predicted to be: a major pilus subunit (fimA), a protein of unknown function (fimB), an usher (fimC), a chaperone (fimD), and 2 minor subunit proteins (fimEF). The 6 genes are located between bases 4016890 and 4022860 of the A449 genome (GenBank accession no. NC_009348). The amino acid (aa) sequences have been assigned GenBank accession numbers YP_001143434 to YP_001143439. Table 3 also shows the relatedness of the A. salmonicida Fim proteins to the homologous proteins of another aeromonad, A. hydrophila, and to the Proteus mirabilis Pmf proteins. While Proteus spp. are not closely related to aeromonads, the number and order of the fim and pmf genes are almost identical in these 2 species and the aa sequences are among the most similar in GenBank (Massad \& Mobley 1994).

To investigate the role of Type I pili in Aeromonas salmonicida pathogenesis, an unmarked deletion mutant strain of A449 was successfully created in which the entire locus fim $A B C D E F$ was deleted. There were no differences in in vitro growth characteristics between the parental A449 strain and the $\Delta$ fim strain (data not shown).

\section{Challenge}

Challenge data are summarized in Table 4. When administered by injection there were no significant differences in overall cumulative morbidity between the $\Delta$ fim mutant strain $(50.0 \%)$ and the parental strain A449 (42.4\%; G-test, p > 0.05). Similarly there were no significant differences when $\Delta$ fim $(45.1 \%)$ and A449

Table 3. Predicted gene products of the Aeromonas salmonicida subsp. salmonicida strain A449 Fim pilus system

\begin{tabular}{|llll|}
\hline \multirow{2}{*}{$\begin{array}{c}\text { A. salmonicida gene name } \\
\text { and GenBank accession no. }\end{array}$} & $\begin{array}{c}\text { A. hydrophila } \\
\text { ATCC 7966 }\end{array}$ & $\begin{array}{c}\text { Homologue GenBank accession no. (\% identity) } \\
\text { Proteus mirabilis }\end{array}$ & \begin{tabular}{l} 
Predicted function \\
\cline { 2 - 4 }
\end{tabular} \\
\hline fimA, YP_001143434 & YP_855052 (93) & pmfA Q04681 (27) & Major pilus subunit \\
fimB, YP_001143435 & YP_855053 (61) & $\begin{array}{l}\text { Not present } \\
\text { pmfC P53514 (44) }\end{array}$ & Unknown \\
fimC, YP_001143436 & YP_855054 (86) & pmfD P53520 (44) & Outer membrane usher \\
fimD, YP_001143437 & YP_855055 (86) & pmfE P53522 (28) & Minor subunit, putative tip adhesin \\
fimE, YP_001143438 & YP_855056 (63) & pmfF P53521 (24) & Minor subunit \\
fimF, YP_001143439 & YP_855057 (75) & & \\
\hline
\end{tabular}


$(44.1 \%)$ were administered by immersion (G-test, p > 0.05). The MTTM of A449 (14.3 d) was significantly shorter than the MTTM for the $\Delta$ fim strain (20.7 d) when administered by immersion, but MTTM was equal at $9.9 \mathrm{~d}$ in both strains when administered by injection.

\section{Stress test}

Six days after the initiation of the stress test, Aeromonas salmonicida-related cumulative morbidity was $75 \%$ in the A449-exposed group and $66.7 \%$ in the $\Delta$ fim group (Table 5). This difference was not significant ( $G$-test, p > 0.05). MTTM was significantly longer in the $\Delta$ fim group $(5.0 \mathrm{~d})$ than in the A449-exposed group (4.2 d; t-test, $\mathrm{p}<0.001)$.

Table 4. Summary of live bacterial challenges. Cumulative morbidity is the number of morbid animals/total number of animals; statistical significance was assessed by a $G$-test with significance set at $\mathrm{p}<0.05$. Mean time to morbidity (MTTM) is the arithmetic mean of time of morbidity in days; statistical significance was assessed by a $t$-test with significance set at $p<0.05$. Different superscripted letters denote statistical significance. nd = not determined

\begin{tabular}{|c|c|c|c|c|}
\hline \multirow{2}{*}{ Strain } & \multicolumn{2}{|c|}{ — Intraperitoneal — } & \multicolumn{2}{|c|}{ - Immersion — } \\
\hline & $\begin{array}{c}\text { Morbidity } \\
(\%)\end{array}$ & $\begin{array}{l}\text { MTTM } \\
\text { (d) }\end{array}$ & $\begin{array}{c}\text { Morbidity } \\
(\%)\end{array}$ & $\begin{array}{l}\text { MTTM } \\
\text { (d) }\end{array}$ \\
\hline A449 & $42.37^{a}$ & $9.9^{\mathrm{c}}$ & $44.05^{\mathrm{d}}$ & $14.3^{\mathrm{f}}$ \\
\hline$\Delta$ fim & $50.0^{\mathrm{a}}$ & $9.9^{\mathrm{c}}$ & $45.12^{\mathrm{d}}$ & $20.7^{g}$ \\
\hline PBS & $0^{\mathrm{b}}$ & nd & $0^{\mathrm{e}}$ & nd \\
\hline
\end{tabular}

Table 5. Stress test results. See Table 4 for statistics and abbreviations

\begin{tabular}{|lcc|}
\hline Strain & Morbidity $(\%)$ & MTTM (d) \\
\hline A449 & $75(30 / 40)^{\mathrm{a}}$ & $4.2^{\mathrm{c}}$ \\
Afim & $66.7(26 / 39)^{\mathrm{a}}$ & $5.0^{\mathrm{d}}$ \\
PBS & $0(0 / 40)^{\mathrm{b}}$ & nd \\
\hline
\end{tabular}

\section{Ex vivo adherence}

Significantly more A449 (192\%) adhered to the gut squares after $2 \mathrm{~h}$ at room temperature (ca. $20^{\circ} \mathrm{C}$ ) than $\Delta$ fim (12.7\%; t-test, p > 0.05) (Fig. 1). The doubling time of A449 at room temperature is ca. $2 \mathrm{~h}$, accounting for the nearly $100 \%$ increase in titer from the original inoculum over the incubation period. However, the number of adherent bacteria that invaded the gut squares was approximately the same in both strains (A449, 35.4\% and $\Delta$ fim, $50.7 \%$; $t$-test, $\mathrm{p}<0.05$ ).

\section{DISCUSSION}

Host colonization, the adherence of a pathogenic bacterium to the host, is a crucial first step in pathogenesis. The S-layer of Aeromonas salmonicida has been repeatedly demonstrated to be its most important adhesin (Ishiguro et al. 1981, Trust et al. 1983, Garduño et al. 1992, 2000, Kay \& Trust 1997). S-layermediated adherence is reportedly against basement membrane components such as Type IV collagen and laminin (Noonan \& Trust 1997). The genome of $A$. salmonicida also carries genes for at least 4 additional
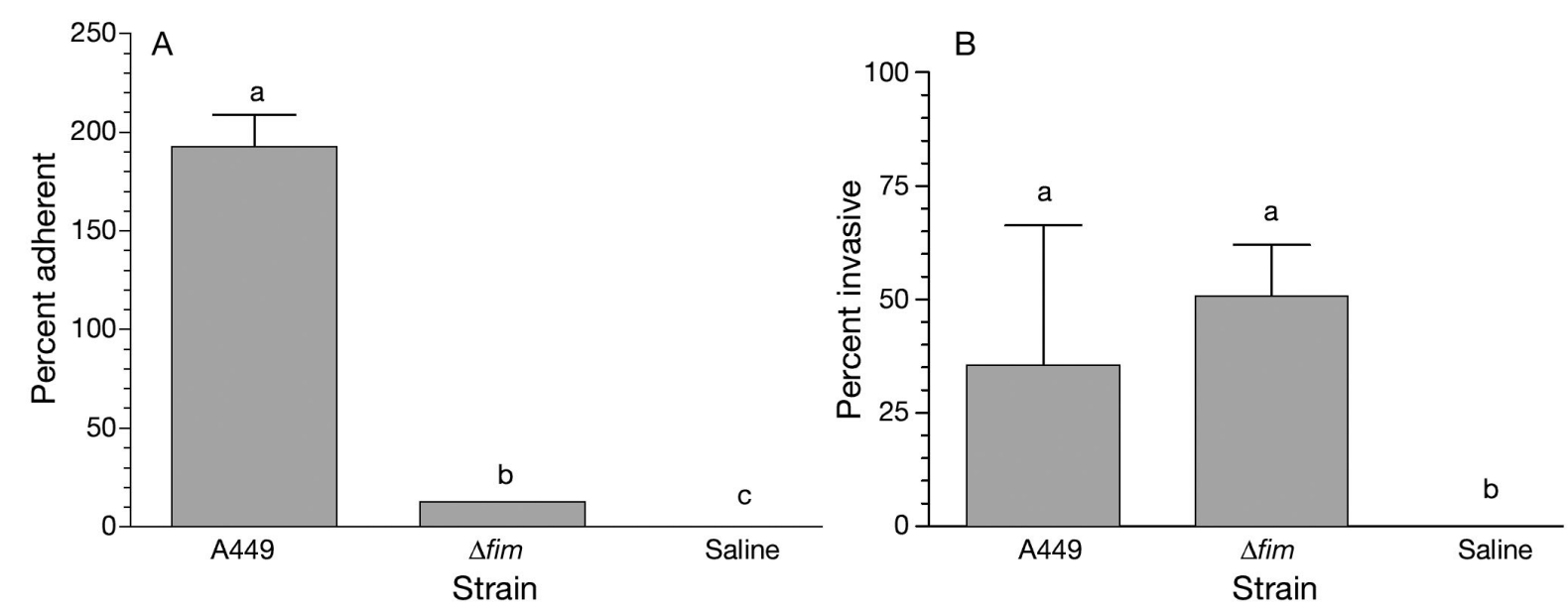

Fig. 1. Contribution of the Fim pilus system to host-cell adherence and invasion. (A) Aeromonas salmonicida strain A449 and the isogenic mutant strain $\Delta$ fim were allowed to adhere to freshly excised squares of Atlantic salmon gut and counted after $2 \mathrm{~h}$ incubation. (B) After a further $2 \mathrm{~h}$ incubation in media supplemented with gentamicin, the epithelial layer was lysed by hypotonic shock and invasive bacteria were counted. Different superscripted letters indicate statistical significance $(t$-test, $p<0.05)$. Data are means (+SD) from 3 gut squares 
adhesins: 3 type IV pili systems, Tap (Masada et al. 2002, Boyd et al. 2008), Flp, and MSHA, of which the latter is expected to be non-functional (Boyd et al. 2008); and 1 Type I or chaperone/usher type pilus, Fim (the present study).

The Fim operon contains 6 genes, which are predicted to encode the major pilin subunit protein $(\operatorname{fim} A)$, a gene of unknown function (fimB), an outer membrane usher (fimC), a periplasmic chaperone (fimD), and 2 minor pilus subunits (fimEF). Based on the aa sequence of the outer membrane usher protein (FimC) and gene order, the Aeromonas salmonicida pilus is in the same group $(\pi)$ as the pyelonephritis-associated (P) pili that allow uropathogenic Escherichia coli to adhere to kidney epithelial cells (Nuccio \& Bäumler 2007). $\mathrm{P}$ fimbriae are composed of a rigid helical shaft that ends in a thinner flexible tip fibrillum, and the single adhesive subunit is carried at the distal end of this fibrillum. The A. salmonicida Fim locus does not have a homologue of the tip fibrillum subunit (PapE) and is therefore not expected to have this structure. The adhesive subunits of P pili (PapG) are larger than the structural subunits and have distinctive N-termini that carry the binding site. Because of its size and sequence, the A. salmonicida fimE gene likely encodes this function.

Despite the presence of 3 presumably functional pili loci, Tap and Flp (Boyd et al. 2008) and Fim (the present study), pili have never been visualized directly on the surface of Aeromonas salmonicida. Seroconversion and the expression of an anti-Tap antibody in rainbow trout experimentally infected with $A$. salmonicida strain A450 has been reported (Masada et al. 2002), suggesting that Type IV pili are expressed under certain in vivo conditions. The surface of strain A449 was extensively examined by atomic force microscopy following growth in high-iron and low-iron media and yet neither peritrichous or polar fimbrial structures were seen (Boyd et al. 2008). It may be that pili and fimbriae in A. salmonicida are either short enough that they do not protrude past the crystalline S-layer, or they are only assembled on contact with the host.

The Type 1 or chaperone/usher pili of Gram-negative bacteria contribute to virulence by allowing the bacterium to adhere to host tissues (reviewed in Pizarro-Cerdá \& Cossart 2006). In the present study the contribution of Fim to the virulence of Aeromonas salmonicida was assessed using an isogenic mutant knockout strain with fim $A B C D E F$ deleted $(\triangle$ fim) in live animal challenges and ex vivo adherence and invasion assays. The entire fimABCDEF operon was deleted as the function of all the genes in the fim operon were not fully understood (for example fimB), and deletion of the whole operon ensured that all adhesive elements were absent in the $\Delta$ fim strain.
Atlantic salmon were challenged with bacteria by either immersion or i.p. injection (Table 4). The former route is considered a more authentic route of exposure than the latter, which bypasses the requirement for adherence and invasion. Perhaps unsurprisingly, given that fim purportedly encodes an adhesin, there were no differences in virulence between $\Delta$ fim and the parental strain, as measured by both cumulative morbidity and MTTM when administered by i.p. injection. Surprisingly, when the $\Delta$ fim strain was administered by immersion, cumulative morbidity was also unchanged with respect to the parental strain. There was, however, a significant increase in MTTM from $14.3 \mathrm{~d}$ (A449) to $20.7 \mathrm{~d}$ in the $\Delta$ fim strain. These results suggest that the $\Delta$ fim strain is equally as virulent as the parental strain, but is impaired in its ability to invade the host.

The stress test data also showed no significant difference between the covert infection levels between the parental strain and $\Delta$ fim (Table 5). However, MTTM was again significantly longer for $\Delta$ fim than it was for the isogenic parental strain. Changes in MTTM for $\Delta$ fim could be due to either a decrease in bacteria resident in the covertly infected fish or the requirement for attachment for the resident bacteria. The latter situation might arise if the covert bacteria are resident in the gut and must adhere and invade to cause infection.

The ex vivo assay confirmed the attenuated colonization properties of the $\Delta$ fim mutant. The gastrointestinal tract (GI) tract was chosen as it could be easily dissected and prepared in a sterile manner. Unlike the resident GI flora of the Atlantic salmon, A449 is chloramphenicol-resistant, allowing it to be discriminated easily (data not shown). It was not possible to use skin as, in contrast, many bacterial species present in the resident tank water are chloramphenicol-resistant, making the discrimination of otherwise unmarked Aeromonas salmonicida strains from the environmental background difficult, and it is impossible to adequately sterilize fish skin without destroying its structural integrity. Ex vivo A. salmonicida adherence and invasion in the salmonid GI has been reported (Ringø et al. 2004, Jutfelt et al. 2008) and most aeromonad species (e.g. A. hydrophilia, A. caviae, A. bestiarum) and the related taxon Pleisomonas are GI pathogens. In the adherence assay, 192\% of the added A449 bacteria were adherent after $2 \mathrm{~h}$ at ca. $20^{\circ} \mathrm{C}$. High adherence data are common in $A$. salmonicida studies, as the hydrophobic S-layer tends to drive bacteria onto surfaces. Furthermore the generation time of $A$. salmonicida at these temperatures is on the order of $2 \mathrm{~h}$ (data not shown), accounting for the increase in bacterial titer. The adherence of the $\Delta$ fim mutant strain was significantly lower than that for the parental strain (Fig. 1), indicating that the Fim pili are the major $A$. salmonicida adhesin for this tissue. 
However, when invasion into the gut epithelium was determined, there was no significant difference between the 2 strains, with invasion rates between 35 and $50 \%$ of the adherent bacteria. Thus while the adherent properties of the $\Delta$ fim strain were significantly attenuated, once adhered to the host (presumably using another adhesin or gravity), it was equally as capable of invasion as the parental strain. Invasion of the epithelial layer by Gram-negative bacteria is often mediated by Type III secretion systems (T3SS). Aeromonas salmonicida has a functioning T3SS (Burr et al. 2002, 2003) and we have shown it to be critical for virulence in Atlantic salmon (Dacanay et al. 2006). The results of the present study suggest that the Fim pili allow $A$. salmonicida to come into close contact with the gut epithelium. This in turn would allow the T3SS to activate cell invasion mechanisms and internalize the bacterium, thereby protecting it from the added gentamicin. However, the data presented here only show that the invasive A. salmonicida were protected from the antibiotic and do not allow us to determine if the invasive bacteria were internalized within the GI epithelial cells or were resident in the intracellular space. The results of Jutfelt et al. (2008), however, suggest that once internalized within the epithelial cell, A. salmonicida can translocate across the epithelium to the serosal side of the intestinal wall, from where it can spread systemically.

In summary, the fim pili are major adhesive factors that allow Aeromonas salmonicida to adhere to salmon intestinal epithelial cells. Lack of fim causes only slight reduction in overall virulence however, reinforcing the notion that $A$. salmonicida virulence is complex and multifactorial.

Acknowledgements. We acknowledge the Dalhousie Aquatron team of J. Batt, G. Whynot, S. Fletcher, and D. Lawrence for providing valuable facilities assistance during the challenges and stress-test. We also thank W. W. Kay and the late J. Thornton for the kind gift of Aeromonas salmonicida subsp. salmonicida strain A449 used during the present study. A.D., J.M.B., M.D.F., and L.C.K. were supported by the Genomics and Health Initiative (GHI) from the National Research Council (NRC) of Canada. This is NRC publication no. 51732 .

\section{LITERATURE CITED}

Boyd JM, Dacanay A, Knickle L, Touhami A and others (2008) Contribution of type IV pili to the virulence of Aeromonas salmonicida subsp. salmonicida in Atlantic salmon (Salmo salar L.). Infect Immun 76:1445-1455

Burr SE, Stube K, Wahli T, Frey J (2002) Evidence for a type III secretion system in Aeromonas salmonicida subsp. salmonicida. J Bacteriol 184:5966-5970

Burr SE, Stuber K, Frey J (2003) The ADP ribosylating toxin, AexT, from Aeromonas salmonicida subsp. salmonicida is translocated via a type III secretion pathway. J Bacteriol 185:6583-6591

Dacanay A, Knickle L, Solanky KS, Boyd JM and others (2006) Contribution of the type III secretion system (TTSS) to virulence of Aeromonas salmonicida subsp. salmonicida. Microbiology 152:1847-1856

> Demers G, Griffin G, de Vroey G, Haywood JR, Zurlo J, Bédard M (2006) Harmonization of animal care and use guidelines. Science 312:700-701

Ellis AE, Burrows AS, Stapleton KJ (1988) Lack of relationship between virulence of Aeromonas salmonicida and the putative virulence factors: A-layer, extracellular proteases and extracellular haemolysins. J Fish Dis 11: 309-323

Garduño RA, Lee EJY, Kay WW (1992) S-layer mediated association of Aeromonas salmonicida with murine macrophages. Infect Immun 60:4373-4382

Garduño RA, Moore AR, Olivier G, Lizama AL, Garduño E, Kay WW (2000) Host cell invasion and intracellular residence by Aeromonas salmonicida: role of the S-layer. Can J Microbiol 46:660-668

Hiney M, Smith P, Bernoth EM (1997) Covert Aeromonas salmonicida infections. In: Bernoth EM, Ellis AE, Midtlyng PJ, Olivier G (eds) Furunculosis. Multidisciplinary fish disease research. Academic Press, London, p 54-97

Ishiguro EE, Kay WW, Ainsworth T, Chamberlain JB, Austen RA, Buckley JT, Trust TJ (1981) Loss of virulence during culture of Aeromonas salmonicida at high temperature. J Bacteriol 148:333-340

Jutfelt F, Sundh H, Glette J, Mellander L, Thrandur Björnsson B, Sundell K (2008) The involvement of Aeromonas salmonicida virulence factors in bacteria translocation across the rainbow trout, Oncorhynchus mykiss (Walbaum) intestine. J Fish Dis 31:141-151

Kay WW, Trust TJ (1997) The surface of Aeromonas salmonicida: What does it look like and what does it do? In: Bernoth EM, Ellis AE, Midtlyng PJ, Olivier G (eds) Furunculosis. Multidisciplinary fish disease research. Academic Press, London, p 235-247

Link AJ, Phillips D, Church GM (1997) Methods for generating precise deletions and insertions in the genome of wildtype Escherichia coli: application to open reading frame characterization. J Bacteriol 179:6228-6237

Masada CL, LaPatra SE, Morton AW, Strom MS (2002) An Aeromonas salmonicida type IV pilin is required for virulence in rainbow trout Oncorhynchus mykiss. Dis Aquat Org 51:13-25

> Massad G, Mobley HLY (1994) Genetic organization and complete sequence of the Proteus mirabilis pmf fimbrial operon. Gene 150:101-104

Metcalf WW, Jiang W, Daniels LL, Kim SK, Haldimann A, Wanner BL (1996) Conditionally replicative and conjugative plasmids carrying lac $Z \alpha$ for cloning, mutagenesis, and allele replacement in bacteria. Plasmid 35:1-13

> Michel C (1979) Furunculosis of salmonids: vaccination attempts in rainbow trout (Salmo gairdneri) by formalin killed germs. Ann Rech Vet 10:33-40

> Noonan B, Trust TJ (1997) The synthesis, secretion and role in virulence of the paracrystalline surface protein layers of Aeromonas salmonicida and A. hydrophila. FEMS Microbiol Lett 154:1-7

> Nuccio SP, Bäumler AJ (2007) Evolution of the chaperone/ usher assembly pathway: fimbrial classification goes Greek. Microbiol Mol Biol Rev 71:551-575

> Olivier G (1990) Virulence of Aeromonas salmonicida: lack of relationship with phenotypic characteristics. J Aquat Anim Health 2:119-127 
Pizarro-Cerdá J, Cossart P (2006) Bacterial adhesion and entry into host cells. Cell 124:715-727

Reith ME, Singh RK, Curtis B, Boyd JM and others (2008) The genome of Aeromonas salmonicida subsp. salmonicida A449: insights into the evolution of a fish pathogen. BMC Genomics 9:427-441

Ringø E, Jutfelt F, Kanapathippillai P, Bakken Y and others (2004) Damaging effect of the fish pathogen Aeromonas salmonicida ssp. salmonicida on intestinal enterocytes of Atlantic salmon (Salmo salar L.). Cell Tissue Res 318: 305-311

Editorial responsibility: David Bruno,

Aberdeen, UK
Schotts EB (1994) Furunculosis. In: Thoesen JC (ed) Suggested procedures for the detection and identification of certain finfish and shellfish pathogens, Version 1, 4th edn, Vol 1. American Fisheries Society, Bethesda, MD

Specker JL, Portesi DM, Cornell SC, Veillette PA (1994) Methodology of implanting cortisol in Atlantic salmon and effects of chronically elevated cortisol on osmoregulatory physiology. Aquaculture 121:181-193

Trust TJ, Kay WW, Ishiguro EE (1983) Cell surface hydrophobicity and macrophage association of Aeromonas salmonicida. Curr Microbiol 9:315-318

Submitted: June 29, 2009; Accepted: September 25, 2009 Proofs received from author(s): January 22, 2010 